\title{
Intrusion Detection Framework Using Efficient Spectral Clustering Technique
}

\author{
K.Vengatesan ${ }^{\mathrm{a}, 1}$, Abhishek Kumar ${ }^{\mathrm{b}}$, K. Harish Eknath ${ }^{\mathrm{a}}$, Sayyad Samee ${ }^{\mathrm{c}}$, Rajiv \\ Vincent $^{\mathrm{d}}$ V.D.Ambeth Kumare \\ ${ }^{a}$ Computer Engineering Department, Sanjivani College Of Engineering, India \\ ${ }^{b}$ Department of Computer Science, Banaras Hindu University, Varanasi, India \\ ${ }^{c}$ Al Sadara, Al Ain, UAE \\ ${ }^{d}$ School of Computer Science and Engineering, VIT, Chennai, India \\ ${ }^{e} \mathrm{CSE}$, Panimalar Engineering College, Chennai, India
}

\begin{abstract}
Developing cyber-security threats are an industrious test for system managers and security specialists as new malware is persistently cleared. Attackers may search for vulnerabilities in commercial items or execute advanced surveillance crusades to comprehend an objective's network and assemble data on security items like firewalls and intrusion detection/avoidance systems (network or host-based). Numerous new assaults will in general be changes of existing ones. In such a situation, rule-based systems neglect to detect the assault, despite the fact that there are minor contrasts in conditions/credits between rules to distinguish the new and existing assault. To detect these distinctions the IDS must have the option to disconnect the subset of conditions that are valid and foresee the feasible conditions (not the same as the first) that must be watched. We have given various techniques to detect intrusions (or anomalies) which are dissipated consistently and structure little clusters of irregular data. To improve the clustering results, the dissipated anomalies are detected and expelled before agent clusters are framed utilizing SC (spectral clustering). For assessment, a manufactured and genuine data set are utilized and our outcomes show that the utilization of SC (spectral clustering) is a promising way to deal with the advancement of an Intrusion Detection System.
\end{abstract}

Keywords. Spectral Clustering, Intrusion Detection System, Anomalies.

\section{Introduction}

Digital security threats are continually developing as foes plan better approaches to vanquish existing systems. These threats are of two primary sorts: ones that utilization parts of known threats and incorporate them to make "another" assault and zero-day 1 assaults where the attacker finds another defenselessness in the item/system that can be abused before it tends to be fixed up. In spite of the fact that detecting zero-day assaults is a perfect desire, as a general rule recognizing assaults that are slight adjustments of existing assaults can be troublesome as well. In this manner, Intrusion Detection Systems (IDS) must be consistently refreshed with the most recent assaults despite the fact that assault designs contrast in just little manners. Consider a case of the Wannacryransomware assault. This malware focused on machines that worked on a more seasoned rendition of Microsoft Windows utilizing a realized endeavor called EternalBlue. An examination of Wannacry uncovered it to be like past assaults [5]. The equivalent is valid with another notable ransom ware ExPetr4 and an altered form Bad Rabbit

\footnotetext{
${ }^{1}$ Vengatesan $\mathrm{K}^{\mathrm{a}}$, Computer Engineering Department, Sanjivani college of engineering, India

Email: vengicse2005@gmail.com
} 
An intrusion detection system (IDS) is one of the most rising errands in network availability[11]. Every year, there are loads of network assaults on the planet; therefore, the expense for taking care of these issues is huge. This issue is a test for government/associations yet in addition to people in everyday lives. To secure the PC network system, by and large, a few strategies can be utilized, for example, firewalls, data encryption, or client verification. The firmware is one technique to ensure the system, yet these days, the outer components have risen and immediately gotten famous. One significant strategy for data mining in the intrusion detection issue proposed in the writing is to utilize AI techniques [1-3]. The IDS has observed straightforwardly the network exchanges where every exchange is either ordinary or noxious. The point of IDS is to detect and caution network executives when it detects an exchange that is an assault. For some situation, the IDS can even quickly obstruct the association.

For the most part, data mining task in IDS must detect two sorts of assault including known assaults and exception (irregularity) assaults. For the known assaults, we can utilize a (semi-)directed learning strategy, for example, neural network, bolster vector machine, irregular timberland, choice tree, and innocent Bayes, to make reference to a couple, to develop a classifier from data preparing (named typical/assaults association) [4-7, 9].

Intrusion Detection Systems (IDS) are of three kinds: (i) Signature-based systems where the assault designs [9] are characterized. These systems can't detect zero-day assaults. As new malware is detected, a recreated signature must be intended for each assault (or joined with others relying upon the system). (ii) Machine learning based systems are of two sorts. They detect abuse by characterizing the traffic as vindictive or benevolent. Abnormality detection systems attempt to characterize "typical" conduct for each procedure on a host system or the network. These systems can't detect a particular assault (like EternalBlue) yet can detect if peculiar (not really noxious) execution occurs. The False Alarm Rate (FAR) can be a test with oddity detection systems. (iii) Hybrid systems that join both machine learning models with signature-based systems. While signature-based systems require consistent principle refreshes, a significant test with data-driven strategies is their powerlessness to traffic that is slanted among favorable and pernicious segments. The applicable datasets that are transparently accessible [8], [6] have a higher level of pernicious traffic when contrasted with a live stream where a lopsidedly enormous part of the traffic is benevolent. As depicted above, rule-based systems can't counter threats that go astray from pre-characterized marks. We take care of this issue by building a model that abduces likely missing conditions/predecessors from rules.

\section{Proposed Efficient Spectral Clustering Aggregation Technique}

In perspective on the imbalanced data circulation of network interfacing practices, we propose an Efficient Spectral Clustering Technique (ESCT) in this area. Right off the bat, we can choose each two groups in the intrusion detection information set to do characterization, and group the examples of the main stream class utilizing the standardized cut spectral clustering calculation.

\section{Input: imbalanced training test set in intrusion data set}

$$
\begin{aligned}
& \mathrm{D}=\mathrm{D} 0 \cup \mathrm{D} 1=\{(\mathrm{x} 1,0), \mathrm{i}=1,2,3, \ldots . . \mathrm{n} 0\} \cup\{(\mathrm{x} 1,1), \mathrm{i}=1,2,3, \ldots . . \mathrm{n} 1\}, \\
& \text { where } \mathrm{n} 1<<\mathrm{n} 0
\end{aligned}
$$




\section{Output: the decision rule}

1. $\left[C_{1}, C_{2}, \ldots, C_{n_{1}}\right]=E S C T\left(D_{0}, n_{1}\right)$;

2. $\left[m_{1}, m_{2}, \ldots, m_{m}\right]=$ Mean_computing $\left(\left[C_{1}, C_{2}, \ldots, C_{n_{1}}\right]\right)$;

3. $D_{0}^{\text {new }}=\left\{\left(m_{t}, 0\right), i=1, \ldots, n_{1}\right\}$;

4. $T_{\text {nev }}=D_{0}^{\text {new }} \cup D_{1}$

5. $H=M\left(T_{\text {nass }}\right)$

Figure 1. The proposed ESCT algorithm

The quantity of clusters is equivalent to the quantity of tests in the alternative class. Subsequent to clustering, we figure the mean value of each cluster that indicated as $\mathrm{m} 1, \mathrm{~m} 2, \mathrm{~m} 3 \ldots \mathrm{mk}$, and afterward utilize every one of the way to develop the novel preparing group of the greater part class. At last, we utilize the first sectional class tests and the novelmain stream class tests to prepare a classifier. The ESCT calculation with pseudo-code is given in Figure 1. A delineation on the ESCT calculation is stated in Figure 2, here blue 5-pointed stars with red circle focuses are two classes. The blue 5-pointed stars are the greater part class then the red circle focuses are the sectional class.
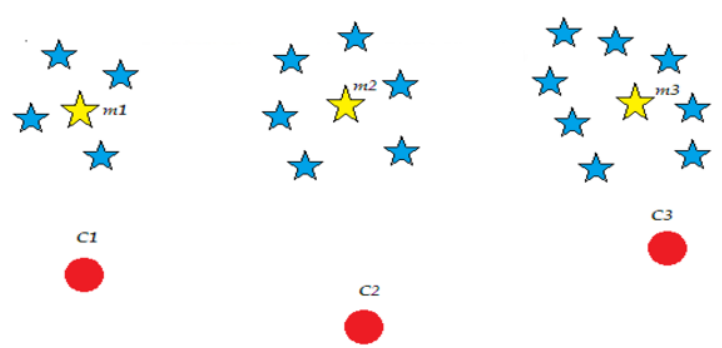

Figure 2. An architecture on the ESCT algorithm.

As appeared in Figure 2, the ESCT calculation cluster the larger part class into three clusters, where the no. 3 equivalents the example count of the sectional class. This 3 clusters are signified by C1, C2 and C3. Since the main stream class tests are supplanted by the methods for every one of the clusters, like $\mathrm{m} 1, \mathrm{~m} 2$, and $\mathrm{m} 3$, the extent of the novel dominant part class are equivalent to that of the sectional class. Along these lines, the ESCT calculation can adequately decrease the awkwardness between various groups. Besides, later $\mathrm{m} 1, \mathrm{~m} 2$, and $\mathrm{m} 3$ are the methods for the clusters, the appropriation data of the main stream class can be saved roughly. Therefore, the proposed ESCT calculation is required to success a decent intrusion detection execution.

\section{Implementation}

\subsection{Portrayal of Data Sets}

The sample data sets are the legitimate testing data sets in present intrusion detection arena. The information are obtained from the DARPA intrusion detection assessment program and comprise of around 5,000,000 info cases. For every TCP/IP association, 
there are 41 measureable and subjective highlights. A few highlights are essential highlights (e.g.: span, convention type and so forth), while different highlights are acquired by utilizing some area information (e.g.: number of fizzled login endeavors and so forth). Among every one of the 41 highlights, there are 8 emblematic highlights and 33 nonstop highlights. Assaults are isolated into four primary classes:

$>$ PROBING, like as port scanning attacks

$>$ User to Root (U2R), like as ejecting attacks

$>$ Denial of Service(DOS), like as ping of death attacks

$>$ Remote to User (R2U), like as guest attacks

So as to fulfill the dual suspicions above, we have to channel the crude preparing data-items. We pick 63808 cases as the preparation set from the crude data. In this preparation set, there are 69930 ordinary cases with 656 assault examples. Table 1 indicates the assaults remembered for the preparation set.

When selecting the sample data sets, we pick 3 gatherings of information by and large, each-one containing 15,000 records. Table 2 indicates the quantity of information in the sampling data sets.

\section{Measuring the performance of Experiment}

False positive and Detection rate, these are standard measurements for assessing intrusion detection, were utilized in our analyses. In the investigation, the DT(detection rate) is characterized as the quantity of intrusion cases detected by the framework isolated by the absolute quantity of intrusion cases displayed in the sample set. The false-positive rate is characterized as the absolute sum of typical occurrences that are erroneously named intrusions separated by the all-out number of ordinary cases.

Table 1. The number of attacks and its type in sampling data item set

\begin{tabular}{|c|c|l|}
\hline Classifications & Attack-No & \multicolumn{1}{c|}{ Attack Name } \\
\hline PROBING & 125 & $\begin{array}{l}\text { Satan(42),ipsweep(30),nmap(19), } \\
\text { portsweep(35). }\end{array}$ \\
\hline R2U & 130 & $\begin{array}{l}\text { imap(1),ftp_write(5),xlock(9), } \\
\text { guess_passwd(31),warezmaster } \\
\text { (29),multihop(18), named (17), } \\
\text { sendmail(17),phf(2), xsnoop(4). }\end{array}$ \\
\hline U2R & 70 & $\begin{array}{l}\text { xterm(15),buffer_overflow(22), rootkit } \\
(13), \text { loadmodule(2), perl(2), ps(16), }\end{array}$ \\
\hline DOS & 285 & smurf(143),neptune(142), \\
\hline
\end{tabular}


Table 2. No. of data in testing info set

\begin{tabular}{|c|c|c|}
\hline Sampling Dataset groups & $\begin{array}{c}\text { No. of normal } \\
\text { data items }\end{array}$ & $\begin{array}{c}\text { No. of } \\
\text { Anomalous Data }\end{array}$ \\
\hline I & 17955 & 2045 \\
\hline II & 18106 & 1894 \\
\hline III & 18454 & 1546 \\
\hline
\end{tabular}

\subsection{Experimental Results}

It very well may be perceived from Table 3 that in the part of detection proportion, ESCT-based upon ID calculation is superior to UC calculation $[7,10]$. The explanation is that UC calculation manages generally average and effectively grouped data, yet those data which are difficult to order can be correctly characterized by ESCT-based upon ID calculation. Accordingly, as we gauge, ESCT-based upon ID technique has preferred execution over unsupervised clustering method in detecting intrusions.

Table 3. Comparison of algorithm's efficiency

\begin{tabular}{|c|c|c|c|c|c|c|}
\hline \multirow{2}{*}{ Algorithm } & \multicolumn{2}{|c|}{ Group 1 } & \multicolumn{2}{c|}{ Group 2 } & \multicolumn{2}{c|}{ Group 3 } \\
\cline { 2 - 7 } & $\begin{array}{c}\text { Detection } \\
\text { rate(\%) }\end{array}$ & $\begin{array}{c}\text { False } \\
\text { positive } \\
\text { rate(\%) }\end{array}$ & $\begin{array}{c}\text { Detection } \\
\text { rate(\%) }\end{array}$ & $\begin{array}{c}\text { False } \\
\text { positive } \\
\text { rate(\%) }\end{array}$ & $\begin{array}{c}\text { Detection } \\
\text { rate(\%) }\end{array}$ & $\begin{array}{c}\text { False } \\
\text { positive } \\
\text { rate(\%) }\end{array}$ \\
\hline \multirow{2}{*}{$\begin{array}{c}\text { Unsupervised } \\
\text { clustering }\end{array}$} & 61.72 & 0.81 & 48.38 & 2.11 & 50.27 & 1.58 \\
\hline \multirow{2}{*}{$\begin{array}{l}\text { Efficient } \\
\text { spectralclustering }\end{array}$} & 78.21 & 0.76 & 74.28 & 0.89 & 74.33 & 0.82 \\
\hline
\end{tabular}

\section{Conclusion}

The analysis result shows that the ESCT-based upon the ID (Intrusion Detection) algorithm is effective for intrusion findings. This algorithm can get a great exactness by joining the UC Intrusion Detection algorithm, and it doesn't depend on named and separated preparing sets. Besides, the algorithm has a decent presentation in finding obscure intrusions. Be that as it may, because of the absence of best strategies in steady spectral clustering algorithm, we needed to execute the whole algorithm in certifiable frameworks. The future work is to locate a gradual spectral clustering algorithm dependent on advancement techniques. 


\section{References}

[1] Ahmad I, Basheri M, Iqbal MJ, Raheem A. Performance comparison of support vector machine, random forest, and extreme learning machine for intrusion detection. IEEE Access, 2018 May; 6 : 33789-33795.

[2] Bai F, Liu XY, Zhang YL, Lang DP. Research on game model of wireless sensor network intrusion detection. In: Proceedings of the 2019 International Conference on Embedded Wireless Systems and Networks, Beijing, China. 2019 Feb;p: 373-378.

[3] Han L, Zhou M, Jia W, Dalil Z, Xu X. Intrusion detection model of wireless sensor networks based on game theory and an autoregressive model. Inform Sciences, 2019 Feb; 476: 491-504.

[4] Kumar N, Akash H, Prataap AR, Srinath G, Mala C. Intelligent intrusion detection system using decision tree classifier and bootstrap aggregation. In: 2018 8th International Symposium on Embedded Computing and System Design (ISED), Cochin, India. 2018 Dec.

[5] Ring M, Landes D, Hotho A. Detection of slow port scans in flow-based network traffic. PLoS ONE, 2018 Sep; 13(9): e0204507.

[6] Shams EA, Rizaner A. A novel support vector machine based intrusion detection system for mobile ad hoc networks. Wireless Networks, 2018 Jul; 24(5): 1821-1829.

[7] Umer MF, Sher M, Bi Y. A two-stage flow-based intrusion detection model for next-generation networks. PLoS ONE, 2018 Jan; 13(1): e0180945.

[8] Yu X, Chu Y, Jiang F, Guo Y, Gong D. SVMs classification based two-side cross domain collaborative filtering by inferring intrinsic user and item features. Knowledge-Based Systems, 2018 Feb; 141: 8091.

[9] Yu X, Jiang F, Du J, Gong D. A cross-domain collaborative filtering algorithm with expanding user and item features via the latent factor space of auxiliary domains. Pattern Recognition, 2019 Oct; 94 : 96- 109.

[10] V.D.Ambeth Kumar, V.D.Ashok Kumar, S.Malathi and P.Jagaeedesh, Intruder Identification using Footprint Recognition with PCA and SVM Classifiers, International Journal of Advanced Materials Research, 2014;.1345: 984-985. 